\title{
Creating a Geriatrics Curriculum in a Continuing Care Retirement Community
}

\author{
Russell S. Blackwelder, MD, MDiv | Melissa Hortman, EdD | Dawn Leberknight | Scott Bragg, \\ PharmD
}

PRiMER. 2018;2:6.

Published: 2/26/2018 | DOI: 10.22454/PRiMER.2018.788040

\section{Abstract}

Introduction: The new Accreditation Council for Graduate Medical Education (ACGME) guidelines for family medicine residencies increased training requirements for caring for older adults. These guidelines prompted changes to the current geriatrics curriculum at the Trident/Medical University of South Carolina (MUSC) Family Medicine Residency Program. Changes to the training requirements and the residency geriatric experiences reflect an increasingly aging population and many unmet needs in caring for older adults.

Methods: To meet accreditation requirements and the needs of our population, the residency program established a new partnership with a continuing care retirement community (CCRC) and hired another provider to coordinate the curriculum. Changes to the curriculum included more time spent in our CCRC, better longitudinal patient visit continuity, a coordinated interprofessional didactic curriculum, more elective opportunities in geriatrics, and online pharmacotherapy quizzes. The curriculum was assessed with a validated 10-question pre/postresident survey.

Results: Resident responses revealed increased comfort in caring for a geriatric population, increased desire to focus on geriatrics in their medical career, and increased participation in the geriatrics track.

Conclusions: With changes in ACGME requirements, family medicine residency programs must develop a comprehensive curriculum to care for an increasing elderly population. The Trident/MUSC Family Medicine Residency provides a model curriculum for other programs seeking to improve training for their residents and meet these requirements.

\section{Introduction}

With the aging of the US population, enhanced geriatrics competencies are needed among family physicians. Changes made to the Accreditation Council for Graduate Medical Education (ACGME) guidelines for family medicine residency programs on July 1, 2014 created new standards, requiring 100 hours (or 1 month) or 125 patient encounters dedicated to the care of the older patient. ${ }^{1}$ Standards also require care across a continuum of sites and a 24-month longitudinal experience at a long-term care facility. ${ }^{1}$

In South Carolina, where our residency is located, the number of people age 85 and over has increased by $41 \%$ from 2000 to 2010 . By 2030, it is estimated that approximately $20 \%$ of people age 85 and over in South Carolina will live in or near Charleston. ${ }^{2,3}$ Continuing care retirement communities (CCRCs) are essential for reaching the aging population with quality health care services, and family medicine physicians provide the first point of medical contact. ${ }^{4}$ With ample literature suggesting the lack of fellowship-trained geriatricians, the need exists for greater 
geriatric training among family medicine residency programs. ${ }^{5-14}$

Family medicine residency programs must alter their curriculum to ensure ACGME standards are met and residents are equipped to work with this population after training. This study addresses a curriculum redesign to meet the new ACGME program requirements and explore the effectiveness of educational efforts at the Trident/Medical University of South Carolina (MUSC) Family Medicine Residency Program.

\section{Methods}

The Trident/MUSC Family Medicine Residency Program has 30 family medicine residents and operates as a partnership between a large academic institution (Medical University of South Carolina) and a community hospital system (Trident Medical Center). The Village at Summerville is a nonprofit 270 bed CCRC, where residents are trained in caring for older adults. Prior to 2015, the residency geriatric experience included only 2 half days per week over 5 weeks with a community preceptor. In 2015, a faculty member was hired to provide clinical services at the CCRC and oversee implementation and enhancement of the residency program requirements. The residency program's curriculum coordinator and clinical pharmacist also contributed to the faculty champion's effort to develop and implement the curriculum.

Changes to the curriculum were based on ACGME requirements and a needs assessment from former graduates. The needs assessment showed many graduates providing care to older adults and a lower percentage of our graduates indicating they were very well prepared to care for older adults compared to graduates from residencies in South Carolina. Curricular changes included a new 5-week geriatric rotation for PGY-2 residents. Care is provided at the CCRC where the attending and resident physicians see patients collaboratively (Table 1). While on the rotation, each resident presents a 30-minute morning report presentation and completes online geriatric pharmacotherapy questions. For residents seeking more experience, there is a geriatrics elective and a geriatrics track, which provide additional learning and scholarly opportunities. In addition, all PGY-2 and PGY-3 residents participated in a longitudinal curriculum where they explored geriatric topics in didactic sessions and then applied what they learned in small group activities with their longitudinal patients (Table 2).

The goals of the new curriculum were to better prepare graduates for caring for older adults, increase interest in geriatric medicine, and meet ACGME requirements. To assess the effectiveness of the rotation, residents completed a validated pre- and post-self-assessment survey on caring for geriatric populations, ${ }^{14}$ geriatric pharmacotherapy questions, and American Academy of Family Physicians (AAFP) reading questions during the rotation. We also implemented an informational survey that asked residents about their interest in and comfort with geriatrics using a 5-point Likert scale, as well as three open-ended questions that assessed changes in resident interest and strengths and weaknesses of the geriatrics curriculum. ${ }^{15}$ Responses to this survey were intended to measure the overall change between resident information prior to participating and after participation in the longitudinal curriculum. Means on linear quantitative survey items were assessed for differences via one-way analysis of variance (ANOVA) procedures. The open-ended questions were analyzed via standard thematic analysis techniques. ${ }^{16}$

The Trident Medical Center Institutional Review Board approved this study (IRB protocol \#2232) as exempt research. We collected data using REDCap, then imported it into SPSS, a statistical software program, to identify trends. Results are displayed in descriptive statistics including measures of central tendencies and measures of variability.

\section{Results}

The PGY-2 cohort consisted of 10 residents. All residents completed the geriatric rotation during their second year and completed 18 months (out of the 24 required) of the longitudinal geriatric experience.

Nine residents (90\%) completed a 25 Yes/No pre- and post-self-assessment rotation survey showing an absolute $36 \%$ increase in confidence $(P<0.05)$ from the beginning of the rotation to the end (Table 3$)$. Approximately $25 \%$ of the questions had over a $50 \%$ increase in confidence.

All PGY-2 residents (100\%) completed the three pharmacotherapy quizzes along with the rotation exam consisting 
of questions from AAFP readings on geriatric topics during their 5-week rotation. Average scores ranged from $91 \%$ to $97 \%$ correct (Table 4 ).

Nine PGY-3 residents (90\%) completed a follow-up survey that asked their level of agreement with four statements on a 1 to 5 Likert scale (Table 5). Combined, these results showed a 0.89 point improvement in Likert scale responses $(P<0.05)$. Resident qualitative responses revealed themes of increased comfort and interest in caring for the geriatric population, increased desire to focus on geriatrics, and increased participation in the geriatrics track.

\section{Conclusions}

With an increasing number of older adults and longer life spans, there is increased importance to prepare physicians who are able to care for elderly populations. It is imperative that family medicine residency programs implement a comprehensive curriculum that increases resident interest and comfort with geriatric medicine. Our curriculum redesign focused on ACGME requirements and offered a 5-week geriatric rotation, established 24 months of longitudinal experiences at a long-term care facility, and included practical didactic sessions where residents practiced topics they learned with their longitudinal patients. Our curriculum redesign improved resident confidence in providing care to older adults and increased resident interest in geriatric medicine.

Limitations with our study include results obtained from only one study site, small numbers of residents completing the program, and no long-term information on how this curriculum impacted resident interest, or patient outcomes. Results from this analysis need to be repeated in other cohorts and with other residency programs to develop more robust, generalizable findings. Moreover, since no power analysis was completed it is unclear if enough participants were included to detect definitively meaningful improvements in confidence. Future considerations can include measurement and tracking of residents entering geriatric fellowship and impact on resident knowledge in geriatrics through examinations such as the In-Training Examination. ${ }^{17}$

The Trident/MUSC Residency Program serves as a potential model for other residency programs to help meet accreditation requirements and to promote improved interest in geriatric care, as demonstrated by this small study. Future studies should attempt to show how these types of curricular changes impact long-term resident interest in caring for older adults and how improved interest in geriatric medicine impacts patient care outcomes.

\section{Tables and Figures}

Table 1: PGY-2 Geriatric 5-Week Rotation

\begin{tabular}{|c|c|c|c|c|c|}
\hline & Monday & Tuesday & Wednesday & Thursday & Friday \\
\hline AM & Geriatrics & Geriatrics & Geriatrics & Geriatrics & Geriatrics \\
\hline PM & Resident clinic & Resident clinic & Resident clinic & Scholarly activity & Resident clinic \\
\hline
\end{tabular}

Half morning spent in skilled nursing/long-term care, half morning in clinic with assisted living/independent living. 
Table 2: Geriatric Longitudinal Curriculum Topics

\begin{tabular}{|l|l|}
\hline \multicolumn{1}{|c|}{ Medical Care Focused } & \multicolumn{1}{c|}{ Health Care Systems Focused } \\
\hline Dementia care and cognitive assessments & Health systems for older adults \\
Delirium & How Medicare works \\
Movement disorders & Polypharmacy, the Beers Criteria, and deprescribing \\
Sarcopenia and frailty & Geriatric community resources \\
Preventative care for older adults & Advance care planning led by the county \\
Wound care & Probate court \\
Chronic pain and the elderly & Falls assessments and safety planning \\
Chronic conditions in older adults & \\
\hline
\end{tabular}

Table 3: Resident Confidence Before and After Geriatrics Rotation ( $0=\mathrm{No}, 1=\mathrm{Yes})$

\begin{tabular}{|c|c|c|c|c|}
\hline & Mean & SD & Difference & $P$ Value \\
\hline Prerotation survey & 0.53 & \pm 0.25 & \multirow{2}{*}{+0.36} & $<0.05$ \\
\hline Postrotation survey & 0.89 & \pm 0.13 & & \\
\hline
\end{tabular}

Table 4: PGY-2 Scores for Pharmacotherapy and AAFP Quizzes (100-Point Maximum Score)

\begin{tabular}{|c|c|c|}
\hline Pharmacotherapy Quiz & PGY-2 Average & SD \\
\hline Wound care management & 92.26 & \pm 10.06 \\
\hline Dementia & 93.45 & \pm 8.32 \\
\hline Parkinson's disease & 96.92 & \pm 5.22 \\
\hline AAFP Readings Quiz & & \pm 5.9 \\
\hline Geriatrics rotation exam & 90.54 & \\
\hline
\end{tabular}

Table 5: Geriatrics Curriculum Resident Survey Before and After Participating Responses (0=Strongly Disagree, 1=Disagree, 3=Neutral, 4=Agree, 5=Strongly Agree)

\begin{tabular}{|c|c|c|c|c|c|c|}
\hline & $\begin{array}{l}\text { Prior to } \\
\text { Mean }\end{array}$ & $\begin{array}{l}\text { Prior } \\
\text { to SD }\end{array}$ & $\begin{array}{l}\text { After } \\
\text { Mean }\end{array}$ & After SD & $\begin{array}{c}\text { Overall } \\
\text { Difference }\end{array}$ & P Value \\
\hline Interested in learning about geriatrics & 4.77 & \pm 0.67 & 5.00 & \pm 0 & & \\
\hline $\begin{array}{l}\text { Comfortable in caring for the geriatric } \\
\text { population }\end{array}$ & 3.67 & \pm 2.18 & 5.00 & \pm 0 & & \\
\hline $\begin{array}{l}\text { Planning to focus on geriatrics in my } \\
\text { medical career }\end{array}$ & 2.44 & \pm 2.01 & 3.44 & \pm 2.13 & & \\
\hline Join the geriatrics track & 2.11 & \pm 2.15 & 3.11 & \pm 2.42 & & \\
\hline Average Total Score & 3.25 & & 4.14 & & +0.89 & $<0.05$ \\
\hline
\end{tabular}

\section{Acknowledgments}

Parts of this study were presented at the 2016 STFM Annual Spring Conference, April 30-May 4, in Minneapolis, MN, and the 2015 STFM Annual Spring Conference, May 3-7, in Orlando, FL. 


\section{Corresponding Author}

Russell S. Blackwelder, MD, MDiv

Medical University of South Carolina, Department of Family Medicine, 9228 Medical Plaza Drive, Charleston, SC 20406. 843-876-7080. Fax: 843-876-7111.

blackwr@musc.edu

\section{Author Affiliations}

Russell S. Blackwelder, MD, MDiv - Trident/MUSC Family Medicine Residency, Medical University of South Carolina, Charleston, SC

Melissa Hortman, EdD - Trident/MUSC Family Medicine Residency, Medical University of South Carolina, Charleston, $\mathrm{SC}$

Dawn Leberknight - Trident/MUSC Family Medicine Residency, Medical University of South Carolina, Charleston, SC Scott Bragg, PharmD - Trident/MUSC Family Medicine Residency and Clinical Pharmacy and Outcome Sciences, Medical University of South Carolina, Charleston, SC

\section{References}

1. Accreditation Council for Graduate Medical Education. ACGME Program Requirements for Graduate Medical Education in Family Medicine. https://www.acgme.org/Portals/0/PFAssets/ProgramRequirements /120_family_medicine_2016.pdf. Accessed March 24, 2017.

2. South Carolina Revenue and Fiscal Affairs Office. Population projections calculated by South Carolina Department of Revenue and Fiscal Affairs, 2010. http://abstract.sc.gov/chapter14/pop5.html Accessed Februrary 2018.

3. Centers for Disease Control and Prevention. The State of Aging and Health in America 2013. Atlanta, GA: Centers for Disease Control and Prevention; 2013.

4. Miller KE, Zylstra RG, Standridge JB. The geriatric patient: a systematic approach to maintaining health. Am Fam Physician. 2000;61(4):1089-1104.

5. Weiss BD. Geriatrics: in our residents' future whether they know it and like it or not. Fam Med. 2008;40(10):741-743.

6. Raetz J, Unwin B, Andrilla HA. Attitudes and Barriers Encountered in Training Family Medicine Residents in Nursing Home Care: A National Survey of Program Directors: A CERA Study. Fam Med. 2016;48(4):309-313.

7. Besdine R, Boult C, Brangman S, et al; American Geriatrics Society Task Force on the Future of Geriatric Medicine. Caring for older Americans: the future of geriatric medicine. J Am Geriatr Soc. 2005;53(6) (suppl):S245-S256.

8. Geriatric Medicine. A Clinical Imperative for an Aging Population. New York: American Geriatrics Society and Association of Directors of Geriatric Academic Programs; 2004.

9. Alliance for Aging Research. Medical Never-Never Land: Ten Reasons Why America Isn't Ready for the Coming Age Boom. Washington, DC: Alliance for Aging Research; 2002. https://www.agingresearch.org /publications/view/135\#.WolS8ZPwajg. Accessed February 12, 2018.

10. Martin JC, Avant RF, Bowman MA, et al; Future of Family Medicine Project Leadership Committee. The Future of Family Medicine: a collaborative project of the family medicine community. Ann Fam Med. 2004;2(suppl

1):S3-S32.

https://doi.org/10.1370/afm.130.

11. Medina-Walpole A, Barker WH, Katz PR, Karuza J, Williams TF, Hall WJ. The current state of geriatric medicine: a national survey of fellowship-trained geriatricians, 1990 to 1998. J Am Geriatr Soc. 2002;50(5):949-955. https://doi.org/10.1046/j.1532-5415.2002.50225.x.

12. Reuben DB, Zwanziger J, Bradley TB, et al. How many physicians will be needed to provide medical care for older persons? Physician manpower needs for the twenty-first century. J Am Geriatr Soc. 1993;41(4):444-453. https://doi.org/10.1111/j.1532-5415.1993.tb06955.x.

13. Adelman RD, Fields SD, Jutagir R. Geriatric education. Part II: the effect of a well elderly program on medical 
student attitudes toward geriatric patients. J Am Geriatr Soc. 1992;40(9):970-973.

https://doi.org/10.1111/j.1532-5415.1992.tb01998.x.

14. White HK, Buhr G, McConnell E, et al. An advanced course in long term care for geriatric medicine fellows. J Am Med Dir Assoc. 2013;14(7):499-506.

https://doi.org/10.1016/j.jamda.2013.02.012.

15. Blackwelder R, Hortmann M, Leberknight D, Bragg S. Trident/MUSC FM Geriatric Survey. Retrieved from STFM Resource Library: https://resourcelibrary.stfm.org/viewdocument/tridentmusc-family-medicinereside?CommunityKey=2751b51d-483f-45e2-81de-4faced0a290a\&tab=librarydocuments. Accessed February $12,2018$.

16. Boyatzi RE. Transforming Qualitative Information: Thematic Analysis and Code Development. Thousand Oaks, CA: SAGE Publications Inc; 1998.

17. American Board of Family Medicine. In-Training Examination Website. https://www.theabfm.org/cert/ite.aspx. Accessed January 10, 2018.

Copyright $(\subset 2018$ by the Society of Teachers of Family Medicine 